\title{
The advanced phases of massive stars and the explosive yields
}

\author{
Alessandro Chieffi ${ }^{1} \dagger$ and Marco Limongi ${ }^{2,3,4}$ \\ ${ }^{1}$ Istituto di Astrofisica e Planetologia Spaziale - INAF \\ email: alessandro.chieffi@inaf.it \\ ${ }^{2}$ Osservatorio Astronomico di Roma - INAF \\ email: marco.limongi@oa-roma.inaf.it \\ ${ }^{3}$ Kavli Institute for the Physics and Mathematics of the Universe, Todai Institutes for \\ Advanced Study, The University of Tokyo, Kashiwa 277-8583, Japan \\ ${ }^{4}$ European Southern Observatory, Karl-Schwarzschild-Str. 2, 85748 Garching bei Munchen, \\ Germany
}

\begin{abstract}
I will briefly review the dependence of the explosive yields on the initial mass,
\end{abstract} metallicity and initial rotational velocity.

Keywords. Stars: evolution, Stars: interiors, Stars: rotation, Stars: Supernovae, Stars: abundances

\section{Introduction}

Massive stars play an active and fundamental role in both the physical and chemical evolution of the Galaxies. Among the others, they strongly contribute to the progressive enrichment of the interstellar gas in elements with $Z>=2$. In particular they dominate the production of the intermediate elements $\mathrm{O}$ to $\mathrm{Ca}$, contribute to the synthesis of $\mathrm{C}$, of the Fe-peak nuclei ( $\mathrm{Sc}$ to $\mathrm{Zn}$ ) and of the S-weak component ( $\mathrm{Ga}$ to $\mathrm{Zr}$ ). Viceversa these stars are not supposed to be primary producers of $\mathrm{N}, \mathrm{F}$ and the main $\mathrm{S}$ component. In some specific cases, like at $Z=0$, a consistent amount of $\mathrm{N}$ may be produced as a consequence of the penetration of the He convective shell in the $\mathrm{H}$ rich mantle.

The amount of matter synthesized by each star depends on its initial mass, chemical composition and rotational velocity. In the following we will briefly present our latest grid of models and associated explosive yields. The code will be presented in Sec. 2 while the new yields will be briefly discussed in Sec. 3 .

\section{The FRANEC code}

All present models have been computed with the latest version of the FRANEC evolutionary code whose latest release has been described in Chieffi \& Limongi (2013) and references therein. This version of the code includes the effects of rotation and takes into account two instabilities: meridional circulation and shear. The adopted nuclear network extends from $\mathrm{H}$ to $\mathrm{Bi}$ and follows explicitly the temporal evolution of 335 nuclear species. We added in these computations an additional mass loss when the luminosity exceeds the Eddington one: in particular we assumed that all the mass zones where $L / L_{\text {edd }} \geqslant 1$ are instantaneously lost from the star. The adopted solar chemical composition is the

$\dagger$ Present address: IAPS-via fosso del cavaliere, 100 - 00133 Roma, Italy 
Table 1. Elements assumed to be overabundant at metallicities lower than solar. The overabundances where determined according to Cayrel et al. (2004) and Spite et al. (2005)

\begin{tabular}{lll}
\hline \multicolumn{2}{c}{ overabundance } \\
\hline$[\mathrm{C} / \mathrm{Fe}]$ & $=$ & 0.18 \\
{$[\mathrm{O} / \mathrm{Fe}]$} & $=$ & 0.47 \\
{$[\mathrm{Mg} / \mathrm{Fe}]$} & $=$ & 0.27 \\
{$[\mathrm{Si} / \mathrm{Fe}]$} & $=$ & 0.37 \\
{$[\mathrm{~S} / \mathrm{Fe}]$} & $=$ & 0.35 \\
{$[\mathrm{Ar} / \mathrm{Fe}]$} & $=$ & 0.35 \\
{$[\mathrm{Ca} / \mathrm{Fe}]$} & $=$ & 0.33 \\
{$[\mathrm{Ti} / \mathrm{Fe}]$} & $=$ & 0.23 \\
\hline
\end{tabular}

Asplund et al. (2009) one. At lower initial Fe abundances, i.e. $[\mathrm{Fe} / \mathrm{H}]=-1,-2$ and -3 , some elements are assumed to be overabundant with respect to the solar value, see Table 1. The global metallicities therefore are $Z=1.345 \cdot 10^{-2}, 3.236 \cdot 10^{-3}, 3.236 \cdot 10^{-4}$ and $3.236 \cdot 10^{-5}$ The corresponding adopted initial He abundances are $Y=0.265([\mathrm{Fe} / \mathrm{H}]=0)$, $Y=0.25([\mathrm{Fe} / \mathrm{H}]=-1)$ and $Y=0.24$ for the two lowest ones.

\section{The grid of models and the yields}

We computed a grid of models extending in mass between 13 and $80 M_{\odot}(13,15,20$, $25,30,40,60$ and 80$)$, in metallicity between $[\mathrm{Fe} / \mathrm{H}]=0$ and $[\mathrm{Fe} / \mathrm{H}]=-3(0,-1,-2$ and -3$)$ and for three initial equatorial rotational velocities $v=0,150$ and $300 \mathrm{~km} / \mathrm{s}$. All models where followed from the Hayashi track up to the moment of the core collapse. Figs. 1 and 2 show the logarithm of the net yields in solar masses of the elements included in the network as a function of the initial mass for solar metallicity models. Figs. 3 and 4 show the same quantities for $[\mathrm{Fe} / \mathrm{H}]=-2$. In all Figures, the black lines refer to non rotating models while the red one to models initially rotating at $300 \mathrm{~km} / \mathrm{s}$.

The first thing worth noting in Figs. 1 to 4 is that on average the elemental yields tend to increase with the initial mass at all metallicities. This is true for both the elements produced in the hydrostatic and the explosive burnings. The basic reason is that the convective mixing control both the amount of matter processed by a given burning and the final mass-radius relation. Since the sizes of the convective cores in central $\mathrm{H}$ and He burnings and the $\mathrm{C}$ convective shells usually scale directly with the initial mass (at least until the mass loss is not so efficient to reduce significantly the He core mass), the amount of mass processed by the $\mathrm{H}$, He and $\mathrm{C}$ burnings increases with the initial mass and therefore the products of these burnings as well. In addition to this, since the size of the $\mathrm{H}$ convective core determines also the mass of the He core, which in turn controls the final compactness of the star at the moment of the core bounce, also the final massradius relation is largely controlled by the extension of the $\mathrm{H}$ convective core. Since the amount of mass processed by the explosive burnings scales directly with the final massradius relation, it follows that also the yields of the elements produced in the explosive burnings basically increase with the initial mass of a star. It goes without saying that, given the pivotal role of mixing in the synthesis of many elements, any modification of the border of the unstable areas or the details of the mixing could significantly affect the final yields of many elements. By the way it is clear that we are not considering here the main s-component which is basically synthesized in a radiative environment within the ${ }^{13} \mathrm{C}$ pocket in stars of 1 to $3 M_{\odot}$ (Straniero et al. 1995) as well as the nuclei produced by the r-processes. 

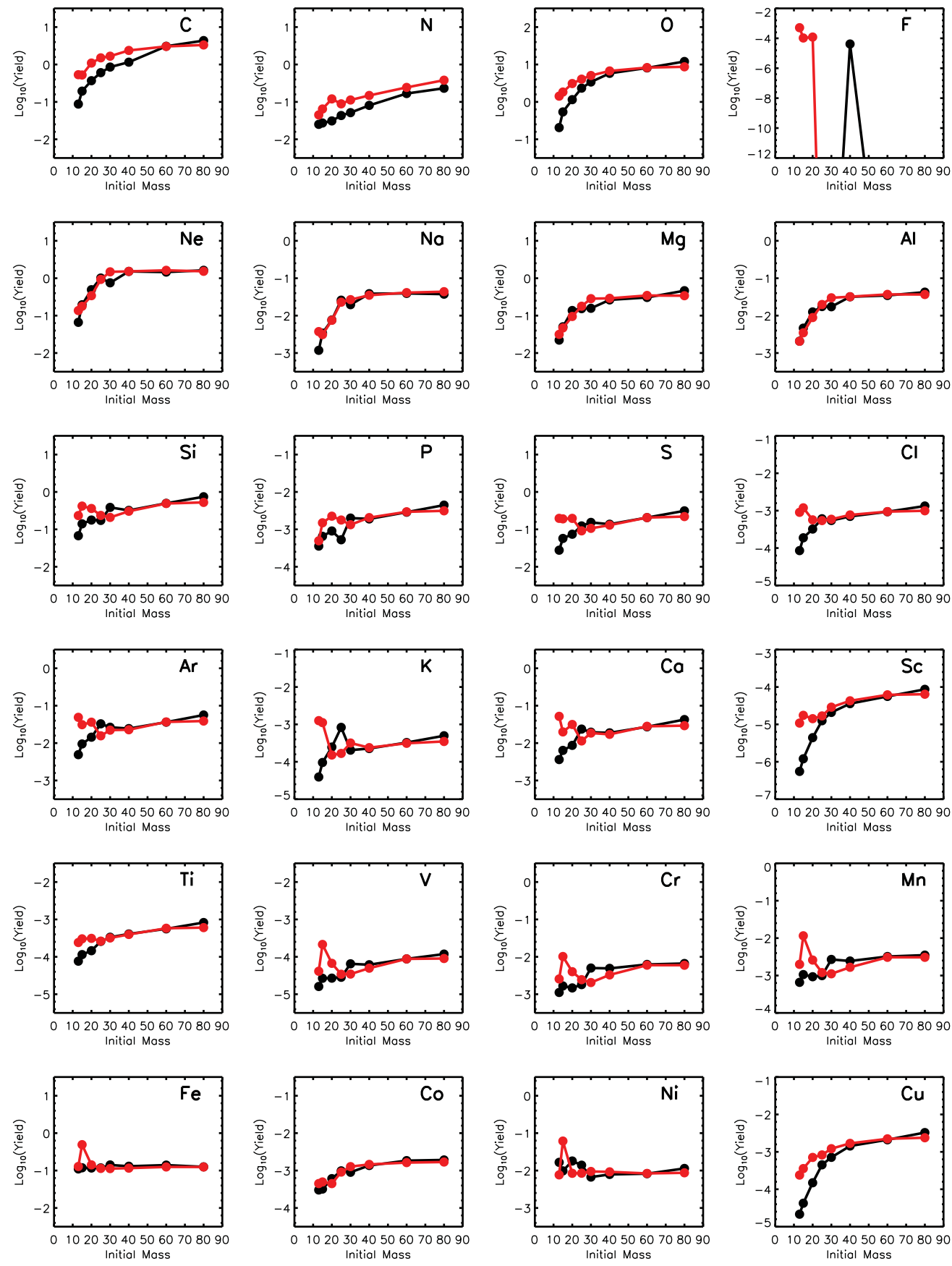

Figure 1. See text.

Since the main effect of rotation is that of favoring an additional mixing that sums to that of the classical thermal instabilities, we can expect that in general the effect of rotation will be that of increasing the yields of the elements. Before proceeding further, it is however important to stress that the amount of mixing induced by the rotational instabilities obviously scales directly with the adopted initial rotational velocity: the 

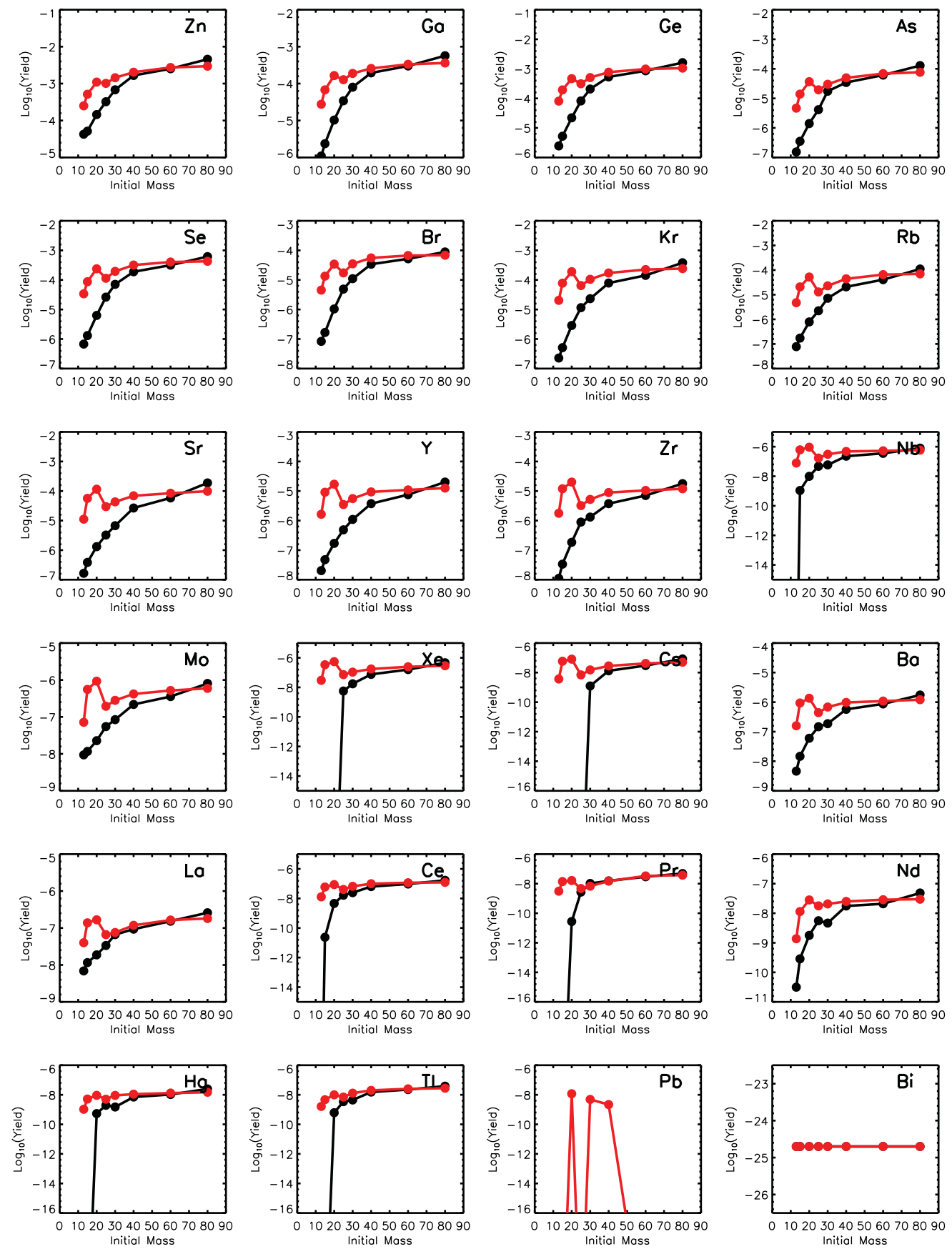

Figure 2. See text.

larger the initial velocity the stronger the effect of the mixing. For sufficiently large initial velocities, the stars can be even forced to be fully mixed and follow an homogeneous evolution (Brott et al. 2011) qualitatively similar to that of very low mass stars $\left(0.5 M_{\odot}\right.$ or less) that are fully convective in central $\mathrm{H}$ burning. For the specific initial rotational velocities chosen here, the influence of rotation on the yields is not very strong, the 

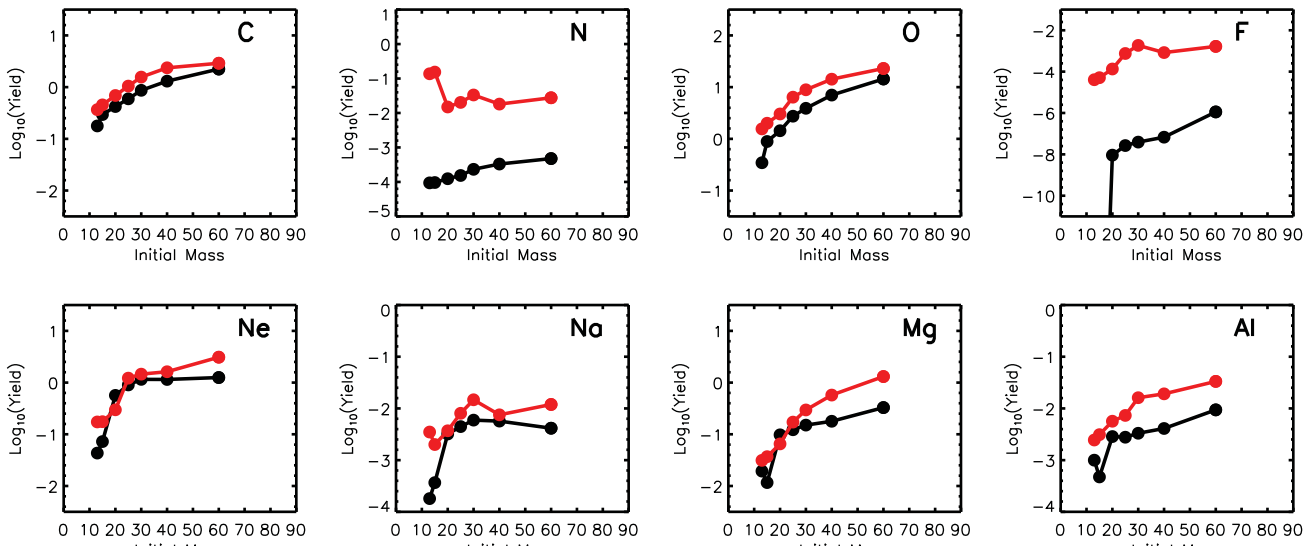

Initiol Moss
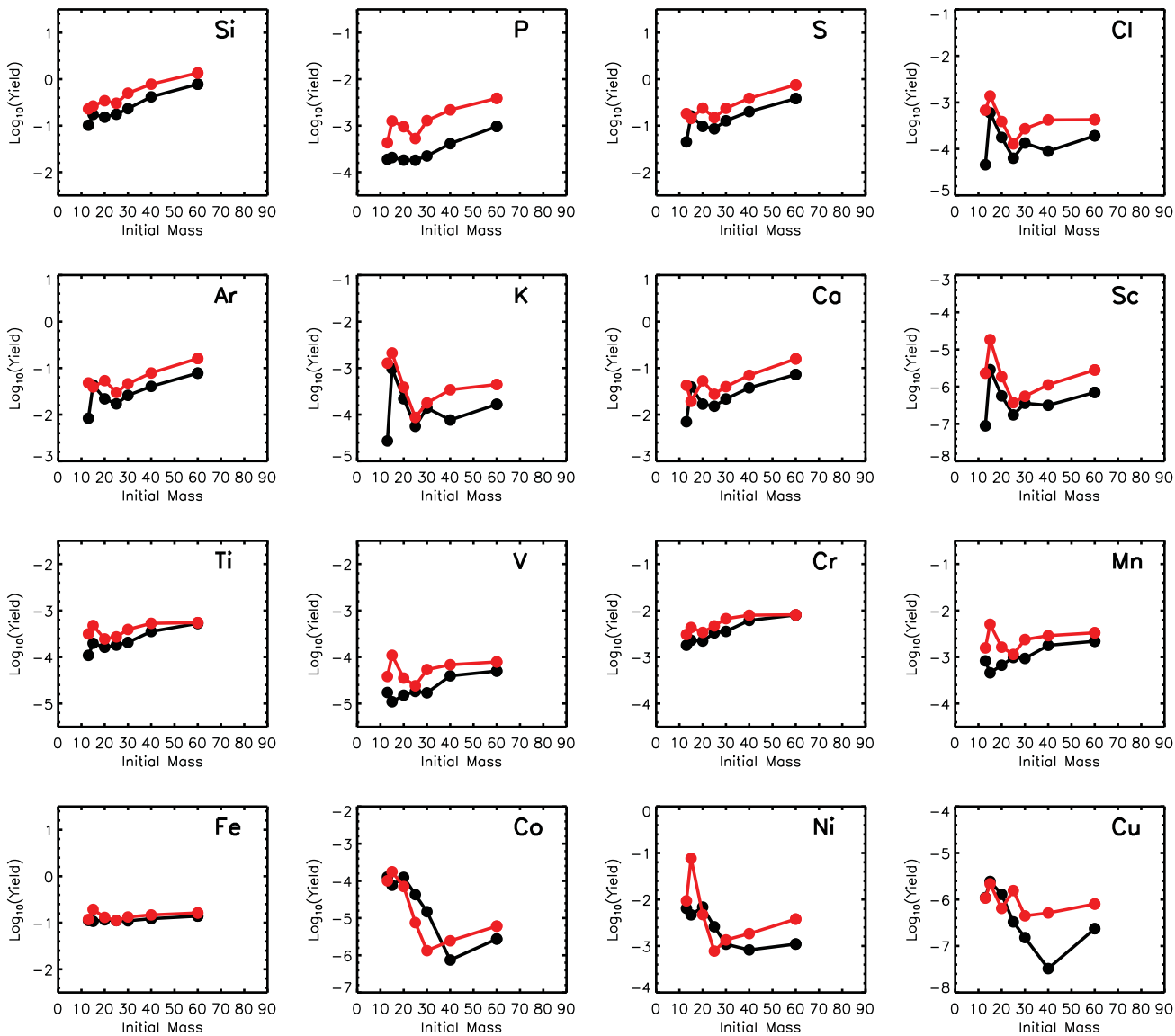

Figure 3. See text.

largest differences occurring for $\mathrm{F}$ and the s-process elements in the sense that rotating models tend to increase significantly the yields of these elements. As we turn to lower metallicities, this effect becomes much stronger, so that at $[\mathrm{Fe} / \mathrm{H}]=-2$, for example, $\mathrm{F}$ and all the s-process elements are largely overproduced with respect to their respective non rotating model. Also $\mathrm{N}$ is largely overproduced by rotating models at sub solar $\mathrm{Fe}$ 

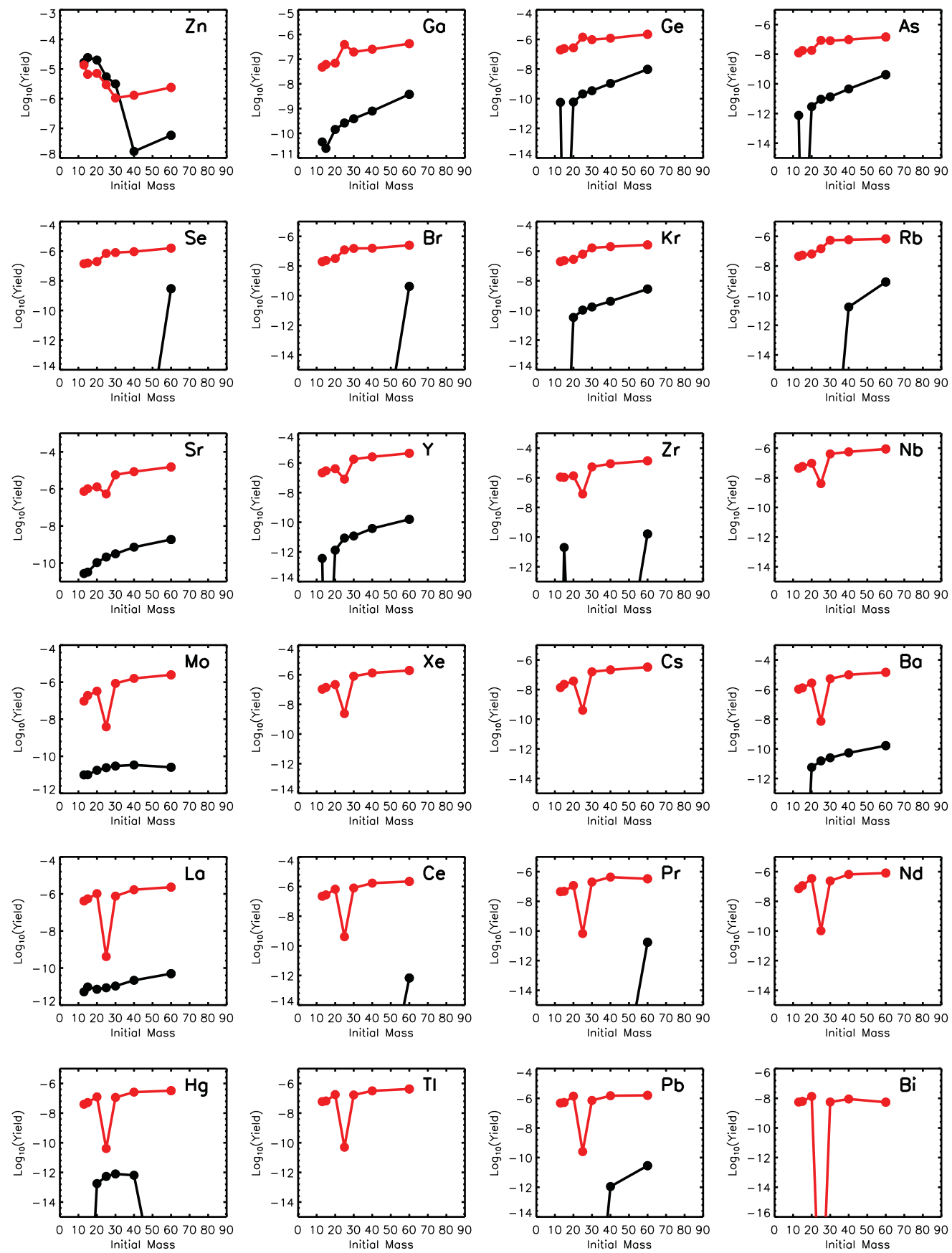

Figure 4. See text.

abundances. We will discuss in detail all such differences in a forthcoming paper. Here, as an example, we will focus only on Fluorine.

In massive stars $\mathrm{F}$ production occurs in the He convective shell that forms after the central He burning in the $\mathrm{H}$ exhausted core, above the outer edge of the He convective core. It is produced by the sequence ${ }^{14} \mathrm{~N}(\alpha, \gamma){ }^{18} \mathrm{~F}(\beta+){ }^{18} \mathrm{O}(p, \alpha){ }^{15} \mathrm{~N}(\alpha, \gamma){ }^{19} \mathrm{~F}$ (Goriely 
et al. 1989; Lugaro et al. 2004). The protons necessary to activate this sequence are produced by the ${ }^{14} \mathrm{~N}(\mathrm{n}, \mathrm{p}){ }^{14} \mathrm{C}$ nuclear reaction that activates also an additional source of ${ }^{18} \mathrm{O}$ through the ${ }^{14} \mathrm{C}(\alpha, \gamma){ }^{18} \mathrm{O}$. The neutron flux may be produced in principle by either the ${ }^{13} \mathrm{C}(\alpha, n){ }^{16} \mathrm{O}$ and/or the ${ }^{22} \mathrm{Ne}(\alpha, n){ }^{25} \mathrm{Mg}$. The nuclear sequence sketched above implies that the key, basic fuel for the $\mathrm{F}$ production is ${ }^{14} \mathrm{~N}$ because it may be either the source of the protons and/or of the neutrons through the ${ }^{22} \mathrm{Ne}$ channel. However, in standard non rotating models ${ }^{14} \mathrm{~N}$ and ${ }^{22} \mathrm{Ne}$ cannot be present simultaneously because, as it is well known, the second one is produced by the burning of the first one. The ${ }^{13} \mathrm{C}$ channel, on the other hand, cannot be efficient because of the low ${ }^{13} \mathrm{C}$ equilibrium abundance left by the CNO cycle in the He core. The only minor production may occur when the He convective shell, after having converted all the ${ }^{14} \mathrm{~N}$ in ${ }^{22} \mathrm{Ne}$, advances in mass engulfing fresh ${ }^{14} \mathrm{~N}$ from the radiative region above it. It goes without saying that this minor production is in any case possible only if the temperature in the He convective shell reaches at least 300 MK. This explains why F is basically destroyed or at most slightly overproduced in massive stars at all (non zero) metallicities. This scenario changes drastically in presence of rotation because of the continuous slow mixing of matter between the He convective core and the active $\mathrm{H}$ burning shell. In particular, freshly produced ${ }^{12} \mathrm{C}$ is continuously brought from the convective core up to the tail of the $\mathrm{H}$ burning shell where it is converted in ${ }^{13} \mathrm{C}$ and ${ }^{14} \mathrm{~N}$ by the $\mathrm{CNO}$ cycle and then spread out within the He core. Vice versa the fresh ${ }^{14} \mathrm{~N}$ brought back in the He convective core is quickly converted in ${ }^{22} \mathrm{Ne}$ and spread out again outside the convective core. The net result is that at the end of the central He burning the abundances of ${ }^{13} \mathrm{C},{ }^{14} \mathrm{~N}$ and ${ }^{22} \mathrm{Ne}$ are all largely enhanced in the He core with respect to the non rotating models. In stars less massive than $25 M_{\odot}$ it is the higher concentration of ${ }^{13} \mathrm{C}$ responsible for the large and systematic $\mathrm{F}$ production. As the initial mass increases the temperature at the base of the convective shell increases as well so that the ${ }^{22} \mathrm{Ne}$ neutron source becomes progressively more important.

All what has been described above does not take into account the fundamental role played by mass loss in the synthesis of $\mathrm{F}$. While the $\mathrm{F}$ net yield increases with the initial mass of the star at $[\mathrm{Fe} / \mathrm{H}]=-2$ (see Fig. 3 ) in both the rotating and non rotating models, at solar metallicity $\mathrm{F}$ is destroyed in the rotating models (for masses larger that $20 M_{\odot}$ ) as well as in the non rotating ones (see Fig. 1). The reason is that, while at $[\mathrm{Fe} / \mathrm{H}]=-2$ mass loss does not fully remove the $\mathrm{H}$ mantle before the end of the central He burning, at solar metallicity mass loss is so efficient that it removes the whole $\mathrm{H}$ rich mantle (and part of the He core as well) well before the central He exhaustion, strongly inhibiting the interplay between the central He burning and the $\mathrm{H}$ shell that is necessary to raise the abundances of the key elements necessary to $\mathrm{F}$ production. By the way, the peak corresponding to the $40 M_{\odot}$ non rotating star visible in Fig. 1 is an artifact due to the fact that this is the only non rotating model that does not destroy F, i.e. for which the net yield is not negative but just slightly positive.

All other features and properties of these models are under analysis and will be presented and discussed in detail as soon as possibile.

\section{Acknowledgements}

A.C. warmly thanks the organizers for the excellent hospitality and for financial support. We acknowledge also the PRIN MIUR 2010-2011 project The Chemical and dynamical Evolution of the Milky Way and Local Group Galaxies, prot. 2010LY5N2T.

\section{References}

Angulo, C., Arnould, M., Rayet, M., et al. 1999, Nuclear Physics A 656, 3 
Asplund, M., Grevesse, N., Sauval, A. J., \& Scott, P. 2009, ARA\& A 47, 481

Brott, I., de Mink, S. E., Cantiello, M., et al. 2011, A\&A 530, A115

Caughlan, G. R. \& Fowler, W. A. 1988, Atomic Data and Nuclear Data Tables 40, 283

Cayrel, R., Depagne, E., Spite, M., et al. 2004, A\&\&A 416, 1117

Chieffi, A. \& Limongi, M. 2013, ApJ 764, 21

Goriely, S., Jorissen, A., \& Arnould, M. 1989, in W. Hillebrandt \& E. Müller (eds.), Nuclear Astrophysics, p. 60

Lugaro, M., Ugalde, C., Karakas, A. I., et al. 2004, ApJ 615, 934

Spite, M., Cayrel, R., Plez, B., et al. 2005, A\&A 430, 655

Straniero, O., Gallino, R., Busso, M., et al. 1995, ApJ (Letters) 440, L85

\section{Discussion}

HiRshI: Which rates are you using for the ${ }^{17} \mathrm{O}(\alpha, \gamma){ }^{21} \mathrm{Ne}$ and the ${ }^{17} \mathrm{O}(\alpha, n){ }^{20} \mathrm{Ne}$ nuclear reactions?

ChIEFf: Caughlan \& Fowler (1988) for the ${ }^{17} \mathrm{O}(\alpha, \gamma){ }^{21} \mathrm{Ne}$ and the NACRE compilation (Angulo et al. 1999) for the ${ }^{17} \mathrm{O}(\alpha, n){ }^{20} \mathrm{Ne}$

Charbonnel: ${ }^{14} \mathrm{~N}$ is a strong poison that captures neutron. Can you comment on its impact on the ${ }^{13} \mathrm{C}$ pocket you produce in the rotating models?

ChIEFf: ${ }^{14} \mathrm{~N}$ plays a fundamental role in the synthesis of Fluorine because it efficiently turns the neutrons produced by the ${ }^{13} \mathrm{C}(\alpha, n){ }^{16} \mathrm{O}$ nuclear reaction in protons necessary to activate the ${ }^{18} \mathrm{O}(p, \alpha){ }^{15} \mathrm{~N}$

MAEDER: Could you please comment on the role of the ${ }^{22}$ Ne pocket as a neutron source in your models?

ChIEFfi: We added this discussion in the text.

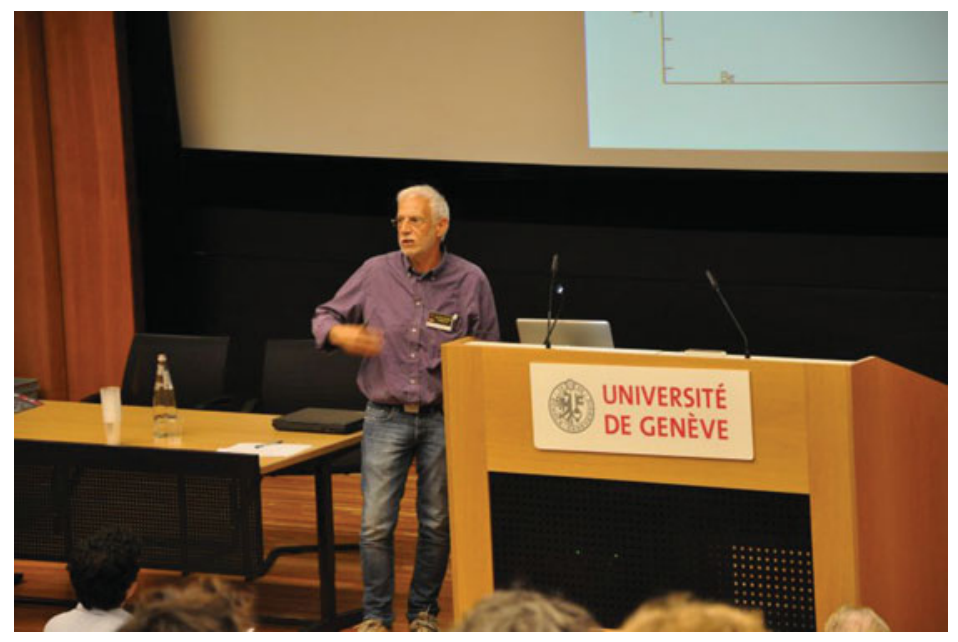

Alessandro Chieffi 